\title{
Eficiência da colagem em madeira de kiri japonês (Paulownia tomentosa)
}

\author{
Efficiency of collage in Japanese kiri wood (Paulownia tomentosa)
}

Fabricio Gomes Gonçalves`, Pedro Gutemberg de Alcântara Segundinho, Juarez Benigno Paes,
Izabella Luzia Silva Chaves, Rhagnya Sharon Ferreira Martins, Sabrina Barros Santiago, Sara Freitas
Souza, Luciana Ferreira Silva, Rafael Gonçalves Espósito Oliveira \& José Geraldo Lima Oliveira

Universidade Federal do Espírito Santo, Jerônimo Monteiro, ES, Brasil. *Autor para correspondência: ufes.fabricio@gmail.com.

Submissão:20/09/2017 / Aceite: 08/08/2018

\begin{abstract}
RESUMO
A colagem em peças de madeira por meio de adesivos é realizada desde a época dos faraós. Com o passar dos anos as tecnologias foram desenvolvidas sobretudo nos adesivos e nas diferentes espécies madeireiras existentes, adaptando-se aos diversos usos pretendidos. Neste contexto também se pode inferir sobre os métodos de análises de sua eficácia. O objetivo desse estudo foi avaliar a resistência na linha de cola mediante o uso de dois adesivos em madeira de Paulownia tomentosa (kiri japonês). As juntas coladas foram preparadas com adesivos resorcinol formaldeído e melamina ureia formaldeído. Avaliou-se a interface madeira-adesivo por meio da espessura da linha de cola principal e secundária com uso de fotomicrografias, resistência ao cisalhamento e percentagem de falha na madeira. Os adesivos apresentaram comportamento desuniforme ao longo da linha da cola. $O$ adesivo resorcinol formaldeído penetrou de forma mais incisiva nos elementos de vasos e raios. A madeira de kiri apresentou percentual de falha superior a $92 \%$, independente do adesivo. A eficiência na colagem com ambos adesivos testados possibilita o uso da madeira de Paulownia tomentosa no Brasil em movelaria e construção civil.
\end{abstract}

PALAVRAS-CHAVE: fotomicrografia, resistência ao cisalhamento, adesivo, linha de adesão.

\begin{abstract}
The collage on pieces of wood using adhesives has been performed since the Pharaoh era. Over the years, technologies have been developed especially for adhesives and different species of wood, adapting to the desired diverse uses. In this context we can also infer the methods of analysis and its efficiency. The aim of this study was to evaluate the resistance in the glue-line by using adhesives in Paulownia tomentosa wood (Japanese kiri). The bonded joints were prepared with resorcinol formaldehyde and melamine urea formaldehyde adhesives. The wood-adhesive interface was evaluated by means of the thickness of the main and secondary glue-lines using photomicrographs, shear strength, and percentage of wood imperfections. The adhesives presented non-uniform behavior in the glue-line. It was possible to observe that the adhesives penetrated in the wood anatomical structures (elements of vessels and rays). The resorcinol formaldehyde adhesive had higher penetration values. The kiri wood imperfection percentage was greater than $92 \%$, independent of the adhesive. The high efficiency observed in bonding with both adhesives allows the use of wood of Paulownia tomentosa in Brazil for furniture and civil construction.
\end{abstract}

KEYWORDS: photomicrographs, shear strength, adhesive, bondline.

\section{INTRODUÇÃO}

A colagem da madeira possibilita um aumento significativo na diversidade de produtos madeireiros, principalmente na fabricação de painéis reconstituídos, portas, janelas, molduras, e em construções estruturais (LIMA et al. 2008). Pesquisas que avaliam a anatomia da madeira na interface com a linha de cola levam a um melhor entendimento, pois fornecem compreensão sobre essa relação, o que é essencial para a redução do consumo de adesivo nas indústrias e principalmente na confecção de produtos de madeira de boa qualidade (ALBINO et al. 2010).

O mecanismo de adesão e a formação de junta colada de qualidade são influenciados pelas 
características anatômicas, químicas, físicas e mecânicas da madeira (GONÇALVES et al. 2016). Outro fator que interfere na colagem é o tipo de adesivo (propriedades físicas e químicas), além disso, deve-se levar em consideração o processo de colagem (ALBINO et al. 2010).

A penetração e distribuição do adesivo nos tecidos anatômicos da madeira, assim como a interação física entre o adesivo e as camadas celulares são muito importantes para o conhecimento dos mecanismos de colagem (SINGH \& DAWSON 2004). As dimensões dos elementos anatômicos, e sua disposição e frequência também influenciam diretamente na colagem, pois estão relacionadas com a porosidade e permeabilidade da madeira (ALBINO et al. 2010).

A madeira de Paulownia tomentosa (Thunb.) Sieb. \& Zucc. ex Steud, conhecida popularmente no Brasil como kiri japonês, é caracterizada por apresentar textura lisa e uniforme, pequena contração e baixa densidade, de 250 a $360 \mathrm{~kg} \mathrm{~m}^{-3}$. Apresenta baixa resistência ao arrancamento de pregos e parafusos, possui uma boa trabalhabilidade, com coeficiente de contração baixo. É resistente a insetos e fungos que causam a podridão branca e parda, o que the confere popularidade no mercado mundial, com uso na indústria moveleira, aviação, em componentes de veículos e navios, além da espécie ser utilizada para a fabricação de painéis de madeira, na forma de compensados, aglomerados e chapas de fibras (CABI 2017, SHU et al. 1986 citado por TANG et al. 2010, PAES et al. 2013). A madeira do gênero Paulownia apresenta raios multisseriados, procumbentes, vasos solitários a múltiplos, difusos e sem obstruções (ZHU et al. 1986).

Os principais adesivos utilizados na indústria madeireira são constituídos a base de formaldeído, como a ureia, a melamina, o fenol, o resorcinol e o isocianato, além do poliacetato de vinila, utilizado pela indústria moveleira e em algumas pesquisas (PIZZI \& MITTAL 2003, BOA et al. 2014, PLASTER et al. 2012 e SEGUNDINHO et al. 2017).

O resorcinol formaldeído é utilizado na fabricação de elementos estruturais e propicia à madeira alta resistência ao cisalhamento, a umidade e às intempéries, com principal aplicação na forma de madeira laminada colada, 'finger joints' e outras estruturas de madeira para usos exteriores (PIZZI \& MITTAL 2003).

O bicomponente melanina ureia formaldeído é um adesivo sintético que possui menor tempo de cura e propicia uma linha de cola transparente (MIOTTO \& DIAS 2009) além de ser mais resistente à umidade quando comparada à ureia formaldeído e ter menor tempo de cura que o fenol formaldeído (IWAKIRI et al. 2005).

Diante do exposto, o objetivo do trabalho foi avaliar o desempenho da madeira colada de kiri japonês com uso dos adesivos estruturais resorcinol formaldeído e melamina ureia formaldeído.

\section{MATERIAL E MÉTODOS}

\section{Origem e preparo do material}

A madeira de P. tomentosa foi disponibilizada pelo Laboratório de Processamento Mecânico da Madeira, do Centro de Ciências Agrárias e Engenharias da Universidade Federal do Espírito Santo.

A madeira foi originária de uma árvore de aproximadamente 26 anos de idade, existente no campus da Universidade, sendo cortada e desdobrada em toras e estas em tábuas, aplainadas e secas ao ar em temperatura ambiente por três meses (umidade de equilíbrio higroscópio de 9,44\%).

Os corpos de provas foram obtidos de uma tábua diametral central para a realização dos ensaios físicos e mecânicos, bem como de resistência da linha de cola, após serem devidamente climatizados a $60 \%$ de umidade relativa e $25 \stackrel{\circ}{\circ}$ de temperatura por um período de quatro semanas.

\section{Ensaios físicos e mecânicos da madeira}

Os ensaios de massa específica aparente, retratibilidade e cisalhamento na madeira de $P$. tomentosa foram realizados de acordo com o Método Brasileiro MB 26 da Associação Brasileira de Normas Técnicas ABNT (1940) (Tabela 1).

\section{Confecção da junta colada}

Após secagem das tábuas, preparou-se lamelas de madeira com dimensões de $22,8 \times 5 \times 2 \mathrm{~cm}^{3}$ (comprimento $x$ largura $x$ espessura). Utilizou-se seis lamelas para confecção de três juntas coladas por tipo de adesivo. Os adesivos utilizados foram resorcinol formaldeído (RF) (Cascophen- RS-216-M) com adição de catalisador (FM-60-M) e melamina ureia formaldeído (MUF) 1242.

A gramatura do adesivo utilizada foi de $300 \mathrm{~g} \mathrm{~m}^{-2}$ aplicado em face dupla. Os adesivos foram espalhados com auxílio de uma espátula sobre a superfície das lamelas, onde sua homogeneidade foi avaliada visualmente e a gramatura controlada por meio de balança digital.

Após aplicação dos adesivos às superfícies da lamela de madeira, as juntas foram unidas e em seguida prensadas a frio em prensa hidráulica com uma pressão de 1,178 $\mathrm{MPa}$ durante 24 horas. 
Confeccionou-se três pares de juntas por tipo de adesivo. As juntas coladas foram acondicionadas em sala de climatização a $25 \pm 2{ }^{\circ} \mathrm{C}$ de temperatura e $60 \pm 2 \%$ de umidade relativa por um período de 20 dias. Posteriormente, foram seccionadas em quatro corpos de prova para o teste de resistência ao cisalhamento, totalizando 12 amostras para cada tipo de adesivo.

Tabela 1. Números de corpos de prova e respectivas dimensões utilizados para os ensaios físico e mecânico na madeira de $P$. tomentosa conforme o método MB 26 (ABNT 1940).

Table 1. Numbers of specimens and their size for the physical and mechanical laboratory tests on $P$. tomentosa wood by the MB 26 method (ABNT 1940).

\begin{tabular}{lcc}
\hline Ensaio & $\begin{array}{l}\text { Número de corpos } \\
\text { de prova }\end{array}$ & $\begin{array}{c}\text { Dimensões } \\
\text { (comprimento x largura x espessura) }(\mathrm{cm})\end{array}$ \\
\hline Massa específica aparente básica & 40 & $3 \times 2 \times 2$ \\
Retratibilidade & 20 & $5 \times 5 \times 5$ \\
Cisalhamento & 24 & \\
\hline
\end{tabular}

\section{Teste de resistência ao cisalhamento e percentual de falhas na linha de cola}

O teste de resistência ao cisalhamento na linha de cola foi realizado de acordo com a norma da American Society for Testing and Materials - ASTM D 905 (ASTM 2009) em uma máquina universal de ensaios com capacidade de $100 \mathrm{kN}$ e obtenção automática dos dados.

O percentual de falha na madeira foi determinado analisando-se visualmente os corpos de provas após ensaio de cisalhamento conforme preconiza a norma ASTM D-3110 (ASTM 1994), a qual exige um valor médio superior a $60 \%$ de falha na madeira.

A resistência foi avaliada pelos critérios da Norma Brasileira Regulamentadora - NBR 7190 (ABNT 1997), que estabelece que a resistência média das juntas coladas deve ser no mínimo igual a resistência característica da madeira ao cisalhamento, no teor de umidade padrão de $12 \%$. Para isto os resultados obtidos nos ensaios foram ajustados para esta umidade (ABNT 1997) (Equação 1):

$$
R_{12}=R_{u \%}\left[1+\frac{3(U \%-12)}{100}\right]
$$

Sendo: $R_{12}=$ valor da resistência a $12 \%$ de umidade; $R_{\cup} \%=$ valor da resistência encontrado no teor de umidade ensaiado; e $U \%$ = umidade do corpo de prova no momento do ensaio.

\section{Análise da espessura da linha de cola por fotomicrografia}

Para análise da interface madeira-adesivo foram retiradas seis amostras centrais referentes a cada tipo de adesivo, com dimensões 1,0 × 1,5 × 2,0 $\mathrm{cm}^{3}$. Estas amostras ficaram submersas em água a temperatura ambiente por 10 dias, para que estas ficassem macias ao corte. Realizou-se o seccionamento dos corpos de prova em micrótomo de deslize, com espessura de $20 \mu \mathrm{m}$. Os cortes obtidos foram fixados em lâminas permanentes com glicerina para obtenção das imagens.

As imagens das lâminas foram obtidas com um microscópio óptico, Axio Scope, modelo A1, utilizando-se a objetiva de $5 \mathrm{X}$ e o filtro 5 , acoplado a um sistema de captura de imagens. As fotomicrografias foram capturadas utilizando-se câmera digital AxioCam MRc5. Utilizaram-se técnicas de microscopia óptica combinada com a microscopia confocal de fluorescência para avaliação da linha de cola das juntas coladas.

Para melhor visualização do comportamento do adesivo na estrutura anatômica da madeira adotouse o filtro de cor azul para as juntas coladas com MUF e o de cor verde para as coladas com RF. Não foram adicionados corantes de qualquer espécie às lâminas.

Para mensuração da espessura média da linha de cola principal realizou-se 40 medições ao longo de cada corte (ALBINO et al. 2010). Já a linha de cola secundária foi quantificada em 15 medições, observando o adesivo que penetrou nas cavidades celulares (vasos e raios). Cada medição foi realizada de uma extremidade a outra na espessura da linha de cola. Utilizou-se o software de análise de imagem AxioVision SE 64 para esta fase.

\section{Análise estatística}

Os dados foram submetidos à análise de variância pelo teste $F$ para verificar se houve diferença $(p<0,05)$ na resistência ao cisalhamento, percentual de falha na madeira e na espessura da linha de cola, principal e secundária entre os dois adesivos utilizados. Anteriormente à ANOVA, foram aplicados aos dados, testes de normalidade (Kolmogorov-Smirnov) e de homogeneidade de variâncias (Kruskal-Wallis), não sendo necessário sua transformação. 


\section{RESULTADOS E DISCUSSÃO}

\section{Características física e mecânica da madeira}

A massa específica aparente básica encontrada para a madeira de $P$. tomentosa apresentou um valor médio de $0,32 \mathrm{~g} \mathrm{~cm}^{-3}$. Espécies pertencentes a este gênero possuem valores de densidade que variam de 0,21 a $0,33 \mathrm{~g} \mathrm{~cm}^{-3}$, o que sugere que o resultado encontrado está em conformidade (ZHU et al. 1986).

A madeira de $P$. tomentosa apresentou um fator anisotrópico médio elevado $(2,32)$, evidenciado pela estabilidade dimensional da madeira a qual está atribuída a grande homogeneidade de seu xilema secundário, refletido nas pequenas variações da densidade $\left(0,30\right.$ a $\left.0,34 \mathrm{~g} \mathrm{~cm}^{-3}\right)$ e das contrações volumétrica $(4,83$ a 5,87\%), tangencial $(2,90$ a 3,96\%) e radial $(1,28$ a 1,72\%). A literatura aponta contração volumétrica inferior $(7,78 \%)$ para $P$. tomentosa. (AKYILDIZ \& KOL 2010).

O ensaio de resistência ao cisalhamento axial da madeira apresentou valores de 5,30 a $6,47 \mathrm{MPa}$, indicando uma baixa resistência mecânica, reflexo da baixa densidade. Dessa forma, esta madeira seria mais indicada para confecção de esquadrias, molduras, forros e outros produtos de uso interno, e não como um componente estrutural.

\section{Avaliação da colagem}

$\mathrm{Na}$ Tabela 2 são apresentados os valores médios e os coeficientes de variação para a espessura da linha de cola principal e secundária, resistência ao cisalhamento e percentagem de falhas na madeira para as juntas coladas de madeira de $P$. tomentosa com os adesivos RF e MUF.

Tabela 2. Valores médios para espessura da linha de cola principal e secundária, resistência ao cisalhamento na linha de cola e falhas na madeira em função do tipo de adesivo nas juntas coladas com madeira de $P$. tomentosa.

Table 2. Average values for line thickness of primary and secondary glue, shear strength and failure wood for the adhesives on joints bonded with $P$. tomentosa wood.

\begin{tabular}{lllll}
\hline \multirow{2}{*}{ Parâmetros } & \multicolumn{4}{c}{ Adesivos } \\
\cline { 2 - 5 } & \multicolumn{3}{c}{ RF } & \multicolumn{3}{c}{ MUF } \\
\cline { 2 - 5 } & Média $^{1}$ & CV $(\%)^{2}$ & Média & CV $(\%)$ \\
\hline Espessura da linha de cola principal $(\mu \mathrm{m})$ & $178,82 \mathrm{a}$ & 23,39 & $148,98 \mathrm{~b}$ & 33,47 \\
Linha de cola secundária $(\mu \mathrm{m})$ & $214,21 \mathrm{a}$ & 35,42 & $124,48 \mathrm{~b}$ & 31,5 \\
Resistência ao cisalhamento da linha de cola $(\mathrm{MPa})$ & $5,42 \mathrm{a}$ & 0,43 & $5,42 \mathrm{a}$ & 21,6 \\
Falha na madeira $(\%)$ & $92,61 \mathrm{a}$ & 5,59 & $92,15 \mathrm{a}$ & 6,88 \\
\hline
\end{tabular}

Mínimo exigido pela Norma ASTM D-3110

$60 \%$ de falha na madeira

${ }^{1}$ Médias seguidas pela mesma letra na linha não diferem estatisticamente $(\mathrm{F}<0,05) .{ }^{2} \mathrm{CV}$ : coeficiente de variação.

A espessura da linha de cola principal variou de 137,0 a $220,64 \mu \mathrm{m}$ para o RF e de 99,12 a 198,84 um para a MUF, indicando a dificuldade de penetração do adesivo na madeira. Os parâmetros espessura de linha de cola principal, e linha de cola secundária apresentaram-se superiores para o adesivo RF, possivelmente devido à viscosidade do mesmo. Embora tenha ocorrido diferença, ao avaliar os parâmetros resistência ao cisalhamento e percentual de falha na madeira pôde-se observar que estes independem do adesivo utilizado.

A resistência ao cisalhamento das juntas coladas foi $5,42 \mathrm{MPa}$, e estas apresentaram elevado percentual de falhas na madeira (92\%) para as resinas RF e MUF (Figura 1), indício de uma boa qualidade da adesão entre as lamelas, com valores superiores ao determinado pela ASTM D-3110, que é de 60\%. Estas duas características são dependentes da superfície das lamelas, da interação entre a madeira e o adesivo e também da presença de tiloses e gomas-resinas (BURGER \& RICHTER 1991, VÁSQUEZ et al. 2003).

A análise estatística demonstrou não haver diferenças significativas entre os tipos de adesivo quanto a resistência ao cisalhamento e o percentual de falhas na madeira. Para a resistência ao cisalhamento, os resultados encontrados foram elevados para os dois tipos de adesivos estudados. $O$ alto percentual de falha na madeira é indicativo de boa qualidade da adesão, pois evidencia que a coesão do adesivo e a resistência da interface adesivo-madeira é igual ou mais elevada que a resistência da própria madeira (VITAL et al. 2005, PLASTER et al. 2012). Além disso, o percentual de falha na madeira pode ser reflexo da formulação do adesivo e das condições ambientais proporcionadas pela colagem (STOECKEL et al. 2013). 

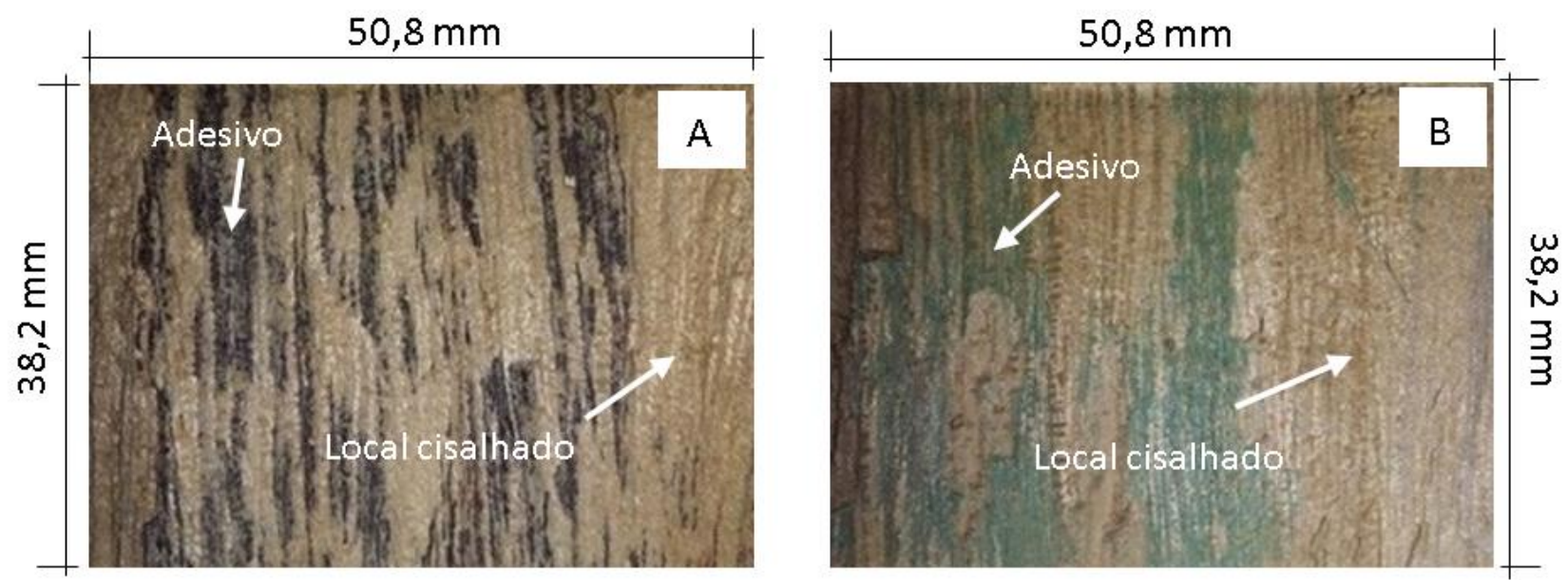

Figura 1. Área cisalhada (A: melamina ureia formaldeído; B: resorcinol formaldeído).

Figure 1. Sheared area (A: melamine urea formaldehyde, B: resorcinol formaldehyde).

Os adesivos PVA e RF, utilizados por VITAL et al. (2006) na colagem com madeiras de Eucalyptus saligna e Eucalyptus grandis apresentaram baixas percentagens de falha na madeira para juntas coladas, variando entre $29,73 \%$ a $41,42 \%$ e $35,69 \%$ a $52,44 \%$ respectivamente. Baixos percentuais de falha na madeira também foram encontrados por IWAKIRI et al. (2013) na avaliação da qualidade das juntas coladas de madeira de eucalipto com o adesivo resorcinol fenol formaldeído, variando de $38,75 \%$ a $63,96 \%$, diferentemente dos valores encontrados no presente estudo.

$O$ alto percentual de falha na madeira (média de $92,38 \%$ ) das juntas coladas pode ser justificado pela baixa densidade da madeira utilizada $\left(0,32 \mathrm{~g} \mathrm{~cm}^{-3}\right)$ e suas características anatômicas. BOA et al. (2014) ao analisarem o comportamento de juntas coladas com ureia formaldeído a partir de resíduos madeireiros de eucalipto verificaram que madeiras de menores densidades, usando a gramatura de $350 \mathrm{~g} \mathrm{~cm}^{-2}$, apresentaram maior qualidade da adesão, maior resistência ao cisalhamento e percentual de falha na madeira quando comparada àquelas com densidade superior.

Uma colagem em planos de corte diferentes também pode interferir na qualidade da adesão na madeira, sendo que naquelas mais densas há maior dificuldade de colagem, em função da dificuldade da penetração do adesivo, resultando em uma junta de qualidade inferior (LOBÃO \& GOMES 2006).

As características das diferentes espécies de madeira podem influenciar na qualidade da colagem, e, portanto, interferir nos resultados de resistência ao cisalhamento e percentual de falha (LIMA et al. 2008).

As propriedades mecânicas de juntas coladas estão associadas às características anatômicas da madeira, devido a uma correlação entre a resistência ao cisalhamento e características como diâmetro do vaso, espessura da parede celular, largura e comprimento da fibra e largura do raio em madeira de eucalipto (ALBINO et al. 2012). Mencionam ainda a existência de correlação entre o percentual de falhas na madeira com a frequência de vaso e altura do raio e com a espessura do lume da fibra.

Pode-se observar que ambos adesivos utilizados apresentaram bons resultados em termos de penetrabilidade na estrutura anatômica (Tabela 2), evidenciado pelo elevado percentual de falha na madeira. Na avaliação do efeito de diferentes gramaturas do adesivo PVA na colagem de taliscas de madeira de teca, FONTE \& TRIANOSKI (2015) observaram que a gramatura não influenciou significativamente na resistência ao cisalhamento, com maior valor absoluto $1,35 \mathrm{MPa}$ na gramatura de 180 $\mathrm{g} \mathrm{m}^{-2}$. BOA et al. (2014) encontraram bons resultados na gramatura de $350 \mathrm{~g} \mathrm{~m}^{-2}$ para grupos de madeira de eucalipto de menor densidade $\left(\leq 0,65 \mathrm{~g} \mathrm{~cm}^{-3}\right)$.

\section{Avaliação da linha de cola por fotomicrografias}

Por meio das fotomicrografias pôde-se observar a interface madeira-adesivo das juntas coladas com os adesivos MUF e RF (Figura 2) na madeira de $P$. tomentosa.

A permeabilidade e porosidade da madeira utilizada permitiram que 0 adesivo penetrasse nas cavidades celulares, resultando em uma linha de cola resistente. Esta boa resistência está associada às características anatômicas da madeira (dimensões, disposição e frequência das células), à densidade, umidade, extrativos e capacidade tampão (URBINATI 2013) (Figura 2). No entanto esta observação deve ser cuidadosa, pois madeiras de maior porosidade e permeabilidade podem gerar linha de cola "faminta" e consequentemente ser menos resistente ao cisalhamento, enquanto madeira de menor permeabilidade na presença de elementos celulares mais complexos, podem formar linha de cola espessa, com dificuldade de penetração do adesivo (IWAKIRI 2005). 

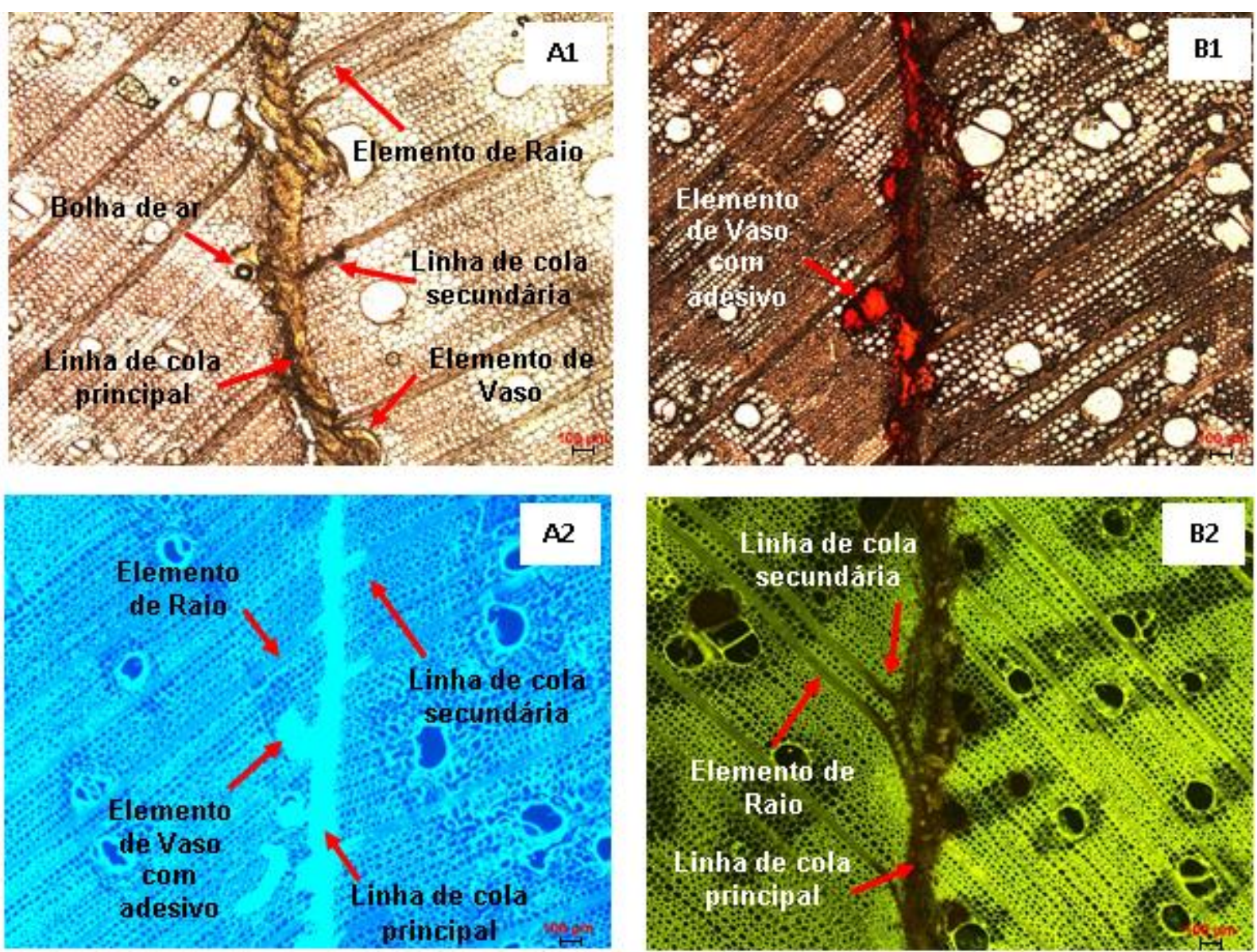

Figura 2. Linha de cola principal e secundária nas juntas coladas com a madeira de $P$. tomentosa (A1 e B1: melamina ureia formaldeído e resorcinol formaldeído com linha de cola observada no microscópio óptico; A2 e B2: melamina ureia formaldeído e resorcinol formaldeído observada na fluorescência com filtros azul e verde. Barra de escala $=100 \mu \mathrm{m}$.

Figure 2. Primary and secondary glue-line in the joints bonded with P. tomentosa wood (A1 and B1: melamine urea formaldehyde and resorcinol formaldehyde with glue-line observed in the optical microscope; A2 and B2: melamine urea formaldehyde and resorcinol formaldehyde observed in fluorescence with blue and green filters. Scale bar $=100$ micrometers.

Pela análise das imagens, foi possível distinguir o adesivo RF na estrutura anatômica da madeira, por apresentar coloração marrom-avermelhada perceptível na linha de cola, principalmente com utilização do filtro verde. Enquanto o adesivo MUF por possuir uma coloração branco leitoso propicia uma linha de cola transparente, atributo considerado importante quando o destino do material é para fins estéticos.

A penetrabilidade do adesivo nas células em decorrência do diâmetro dos vasos e largura dos raios, são determinantes para a aderência entre os substratos (ALBINO et al. 2012). As características anatômicas da madeira de $P$. tomentosa propiciaram a penetração dos adesivos nos elementos celulares, resultando em uma boa aderência do adesivo ao substrato. Os elementos de vaso, raio e parênquima axial são as células envolvidas na ancoragem mecânica do adesivo, bem como pelo espalhamento radial e axial do adesivo (URBINATI 2013).

A penetração do adesivo na madeira também está associada às boas condições de molhabilidade do adesivo (CHANDLER et al. 2005), evidenciando a importância das características do adesivo, como fluidez, transferência e penetração no processo de colagem.

O RF e a MUF apresentaram comportamento desuniforme ao longo da linha de cola, visto que a espessura desta variou na interface madeira-adesivo, devido a possíveis irregularidades na superfície das lamelas, culminando em uma penetração heterogênea de ambos adesivos nos elementos celulares. Alguns autores também relataram que o adesivo RF apresentou o mesmo comportamento, penetrando na madeira e preenchendo cavidades celulares (vasos, raios, parênquimas e fibras) adjacentes a linha de cola principal em madeiras de eucalipto (ALBINO et al. 2010, BIANCHE 2014).

Para obtenção de uma boa colagem é necessário que o intervalo entre a aplicação do adesivo até o 
momento de prensagem seja o menor possível para evitar aumento da viscosidade do adesivo (CUNHA \& MATOS 2010), fator que pode prejudicar sua penetrabilidade na madeira e comprometer o processo de adesão. Neste trabalho, o tempo de aplicação dos adesivos nas faces da madeira de $P$. tomentosa não foi avaliado, no entanto, definiu-se um intervalo de sete minutos entre aplicação do adesivo e a prensagem.

A microscopia permitiu a visualização dos adesivos nas estruturas anatômicas da madeira $P$. tomentosa e avaliação da qualidade de colagem. A importância dessa técnica para observação da linha de cola no processo de adesão é evidenciada por SINGH et al. (2008), ALBINO et al. (2010), BIANCHE (2014), HAN et al. (2016), MODZEL et al. (2011) e URBINATI (2013) pois permite observar o comportamento do adesivo e como este penetra nos elementos celulares.

\section{CONCLUSÃO}

As análises realizadas permitem concluir que: os adesivos penetraram na estrutura anatômica da madeira, adentrando nas cavidades adjacentes à linha de cola principal, preenchendo lúmens de vasos e raios; a qualidade da colagem da madeira de Paulownia tomentosa pode ser atribuída às suas características, como a boa permeabilidade, permitindo uma penetração adequada do adesivo aos elementos celulares; a linha de cola formada pelo RF foi mais perceptível com ajuda da microscopia que a linha de cola da MUF; as fotomicrografias permitiram observar de forma nítida a linha de cola formada, bem como realizar as devidas mensurações; a grande eficiência na colagem com os adesivos RF e MUF permite a fabricação de elementos de madeira laminada colada viabilizando uma utilização da madeira de Paulownia tomentosa no Brasil para movelaria e na construção civil.

\section{AGRADECIMENTOS}

Os autores agradecem ao Conselho Nacional de Desenvolvimento Científico e Tecnológico (CNPq) e à Fundação de Amparo à Pesquisa do Espírito Santo (FAPES) pelo apoio financeiro.

\section{REFERÊNCIAS}

AKYILDIZ MH \& KOL HS. 2010. Some technological properties and uses of paulownia (Paulownia tomentosa Steud.) wood. Journal Environmental Biological 31: 351-355.

ALBINO VCS et al. 2010. Estudo da interface madeira-adesivo de juntas coladas com resorcinol formaldeído e madeira de Eucalyptus grandis W. Hill ex. Maiden. Scientia Forestalis 38: 509-516.

ALBINO VCS et al. 2012. Influência das características anatômicas e do teor de extrativos totais da madeira de Eucalyptus grandis W. Hill ex Maiden na qualidade da colagem. Ciência Florestal 22: 803-811.

ASTM. 1994. American Society for Testing and Materials. Standard specification for adhesives used in nonstructural glued lumber products: ASTM D-3110. Philadelphia: Annual Book of A.S.T.M. Standards. p.184-192.

ASTM. 2009. American Society for Testing and Materials. Standard test method for strength properties of adhesive bonds in shear by compression loading: ASTM D-905. Pennsylvania: Annual Book of A.S.T.M. Standards. p.20-26.

ABNT. 1940. Associação Brasileira de Normas Técnicas. Ensaios físicos e mecânicos da madeira: MB-26. Rio de Janeiro. $16 \mathrm{p}$.

ABNT. 1997. Associação Brasileira de Normas Técnicas. Projeto de estruturas de madeira: NBR 7190. Rio de Janeiro. $107 p$.

BIANCHE JJ. 2014. Interface madeira-adesivo e resistência de juntas coladas com diferentes adesivos e gramatura. Tese (Doutorado em Ciências Florestais). Viçosa: UFV. 85p.

BOA AC et al. 2014. Resíduos madeireiros de eucalipto colados com resina ureia formaldeído à temperatura ambiente. Scientia Forestalis 42: 279-288.

BURGER ML \& RICHTER HG. 1991. Anatomia da madeira. São Paulo: Nobel. 154p.

CABI. 2017. Invasive Species Compendium. Disponível em: http://www.cabi.org/isc/datasheet/39100. Acesso em: 14 ago. 2017.

CHANDLER JG et al. 2005. Examination of adhesive penetration in modified wood using fluorescence microscopy. In: ASC Spring 2005 Convention and Exposition, Maryland, USA. p.17-19.

FONTE APN \& TRIANOSKI R. 2015. Efeito da gramatura sobre a qualidade da colagem lateral da madeira de Tectona grandis. Revista de Ciências Agroveterinárias 14: 224-233.

GONÇALVES FG et al. 2016. Avaliação da resistência ao cisalhamento da madeira de Pinus sp. coladas em temperatura ambiente. Ciência da Madeira 7: 42-50.

HAN J et al. 2016. Interfacial adhesion and damping characteristics of laminated venner intercalated with rubber sheets. Bioresources 11: 6677-6691.

IWAKIRI S. 2005. Painéis de madeira reconstituída. Curitiba: FUPEF. 247p.

IWAKIRI S et al. 2005. Produção de painéis aglomerados de alta densificação com uso de resina melamina-ureiaformaldeído. Cerne 11: 323-328.

IWAKIRI S et al. 2013. Avaliação da resistência de juntas coladas da madeira de Eucalyptus benthamii com diferentes 
adesivos e faces de colagem. Scientia Forestalis 41: 411-416.

LIMA CKP et al. 2008. Colagem da madeira de clones de Eucalyptus com três adesivos comerciais. Scientia Forestalis 36: 73-77.

LOBÃO MS \& GOMES A. 2006. Qualidade da adesão de madeira de eucalipto em corpos-de-prova colados em dois diferentes planos e densidades. Cerne 12: 194-200.

MIOTTO JL \& DIAS AA. 2009. Produção e avaliação de vigas de madeira laminada colada confeccionadas com lâminas de eucalipto. Revista Tecnológica, Edição Especial ENTECA: 35-45.

MODZEL G et al. 2011. Comparative analysis of a wood: adhesive bondline. Wood Science Technology 45: $147-158$.

PAES JB et al. 2013. Efeito de soluções salinas na estabilidade dimensional da madeira de Kiri (Paulownia tomentosa). Revista Científica Eletrônica de Engenharia Florestal 21: 72-84.

PIZZI A \& MITTAL KL. 2003. Handbook of adhesive technology. 2.ed. New York: Marcell Deckker. 999p.

PLASTER OB et al. 2012. Comportamento de adesão da madeira de um híbrido clonal de Eucalyptus urophylla $\times$ Eucalyptus grandis proveniente de três condições de manejo. Ciência Florestal 22: 323-330.

SEGUNDINHO PGA et al. 2017. Eficiência da colagem de madeira tratada de Eucalyptus cloeziana F. Muell para produção de madeira laminada colada (MLC). Revista Matéria 22. 13p.

SINGH A et al. 2008. Light, confocal and scanning electron microscopy of wood-adhesive interface. Microscopy and Analysis 22: 5-8.

SINGH AP \& DAWSON BSW. 2004. Confocal microscope: a valuable tool for examining wood-coating interface. Journal Coatings Technology Research 1: 235-237.

STOECKEL F et al. 2013. Mechanical properties of adhesives for bonding wood - A review. International Journal of Adhesion \& Adhesives 45: 32-41.

TANG ZQ et al. 2010. In vitro induction and identification of tetraploid plants of Paulownia tomentosa. Plant Cell, Tissue and Organ Culture 102: 213-220.

URBINATI CV. 2013. Influência das características anatômicas em juntas coladas de Schizolobium parahyba var. amazonicum (Huber ex. Ducke\} Barneby (paricá). Tese (Doutorado em Ciência e Tecnologia da Madeira). Lavras: UFLA. 161p.

VÁSQUEZ G et al. 2003. Effect of veneer side wettability on bonding quality of Eucalyptus globulus plywoods prepared using a tannin-phenol-formaldehyde adhesive. Bioresource Technology 87: 349-353.

VITAL BR et al. 2005. Efeito de ciclos de umidade relativa e temperatura do ar na resistência de juntas coladas com lâminas de Eucalyptus grandis W. Hill ex Maiden, Eucalyptus saligna Smith e chapas de fibra de densidade média (MDF). Revista Árvore 29: 801-807.

VITAL BR et al. 2006. Qualidade de juntas coladas com lâminas de madeira oriundas de três regiões do tronco de Eucalyptus grandis, Eucalyptus saligna e Pinus elliotti. Revista Árvore 30: 637-644.

ZHU ZH et al. 1986. Paulownia in China: cultivation and utilization. Beijing: Chinese Academy of Forestry. 65p. Disponível em: https://idl-bnc-idrc.dspacedirect.org/bitstream/handle/10625/8226/71235.pdf?seque nce=1. Acesso em: 14 ago. 2017. 